\title{
Minimization of thermal resistance in an air cooled porous matrix made up of solid spheres with heat generation
}

\author{
J. Baloyi, T. Bello-Ochende ${ }^{1}$, J. P. Meyer \\ Department of Mechanical and Aeronautical Engineering, University of Pretoria, Pretoria, Private \\ Bag X20, Hatfield, 0028, South Africa
}

\begin{abstract}
In this paper an analytical model was developed to minimize the thermal resistance of an air cooled porous matrix made up of solid spheres with internal heat generation. This was done under the assumption of local thermal equilibrium. The analytical solution of the optimum sphere diameter was found to be independent of the heat generation rate of the solid spheres, but was dependent on the applied pressure drop and fluid properties. The analytical model compared very well to a numerical model found in a computational fluid dynamics code when air and liquid water properties were used for the fluid phase and wood and silica/sand properties were used for the solid phase.
\end{abstract}

Keywords: porous media, optimization, local thermal equilibrium. Internal heat generation

\section{Nomenclature}

A porous domain cross sectional area, $\mathrm{m}^{2}$

Be Bejan number

$c_{p} \quad$ fluid heat capacity at constant pressure, J/kg.K

D diameter of the solid sphere, $\mathrm{m}$

K $\quad$ porous medium permeability, $\mathrm{m}^{2}$

$K_{f} \quad$ fluid thermal conductivity, W/m.K

$k_{m} \quad$ porous medium thermal conductivity, W/m.K

$k_{\mathrm{s}} \quad$ sphere thermal conductivity, W/m.K

$L \quad$ length of the porous medium, $m$

\footnotetext{
${ }^{1}$ Corresponding author.

E-mail address: Tunde.Bello-Ochende@up.ac.za (T. Bello-Ochende)
} 
$P \quad$ pressure, $\mathrm{Pa}$

$q \quad$ heat transfer rate, $\mathrm{W}$

$q^{\prime \prime \prime} \quad$ heat source density, $\mathrm{W} / \mathrm{m}^{3}$

$q^{\prime \prime}{ }_{w} \quad$ heat flux, $\mathrm{W} / \mathrm{m}^{2}$

$R_{T} \quad$ thermal resistance, $\mathrm{K} / \mathrm{W}$

$T_{\max }$ maximum temperature at the centre of the sphere, $\mathrm{K}$

$T_{\min } \quad$ minimum temperature at the porous medium inlet, $\mathrm{K}$

$T_{\text {outlet }}$ temperature at the porous medium outlet, $\mathrm{K}$

$T_{\text {inlet }} \quad$ temperature at the porous medium inlet, $\mathrm{K}$

$u \quad$ streamwise fluid velocity component, $\mathrm{m} / \mathrm{s}$

$\vec{v} \quad$ Superficial fluid velocity vector, $\mathrm{m} / \mathrm{s}$

$x \quad$ streamwise position variable, $\mathrm{m}$

\section{Greek letters}

$\alpha \quad$ thermal diffusivity

$\gamma \quad$ solid/fluid thermal conductivity ratio

$\eta \quad$ dimensionless thermal resistance

$\mu \quad$ fluid dynamic viscosity, Pa.s

$\phi \quad$ porosity

$\rho \quad$ fluid density, $\mathrm{kg} / \mathrm{m}^{3}$

$\Psi \quad$ dimensionless sphere diameter

$\theta \quad$ dimensionless temperature

\section{Subscript}

coarser coarser grid

finer finer grid

min minimum 
$\max$

maximum

opt optimum

\section{Introduction}

The storage of spent nuclear fuel is complicated by the need to manage the decay heat generated by the fuel. The spent nuclear fuel has to be cooled so that heat generated does not melt the containment system, which could lead to unintentionally release of radioactive material to the surrounding. Another instant where a similar scenario takes place, although innocuous, is whereby a pile of stones (heated a prior as an energy storage mechanism) are cooled by a fluid flowing through gaps between them. The heat transfer mechanisms involved in the cooling in both instances have historically been analysed by assuming that the fluid and solid phases are at local thermal equilibrium (LTE) in order to simplify the analysis. However in general models created with this assumption in mind do not give accurate results because the local temperature difference between the solid and fluid phases can be too big for the LTE assumption to be valid. In order to remedy this shortcoming local thermal nonequilibrium (LTNE) has been taken as the general condition which is assumed to prevail between the solid and fluid phases.

Using the LTNE condition between the two phases many researchers [1-6] created models in an attempt to solve the heat transfer problem for porous media and they showed that these models asymptote into LTE models at special conditions. Meanwhile [7-14] compared the LTNE and LTE models for cases from metallic and non-metallic packed beds to microchannel heat sinks and annulus partially filled with porous media. They come up with criteria under which the LTE assumption was valid. Reddy and Narasimhan [15] numerically investigate a similar case where there is interplay between internal heat generation and externally driven natural convection inside a vertical porous annulus under steady state conditions.

Whitaker [16] discusses constraints that should be satisfied when LTE was assumed. He suggested that these constraints must be satisfied when the following conditions are imposed: the solid and fluid phases' thermal diffusivities ratio must equal, the thermal conductivities ratio must be equal, and the non-slip condition must hold. [17] and [18] developed LTE models for metallic foams porous channels where they respectively discovered that the LTE assumption was valid even if the solid thermal conductivity was markedly higher than that of the fluid, and developed an algorithm that does away with the need to conduct numerical calculations. Alazmi and Vafai [1] investigated the proper boundary condition to be used for a porous channel bounded by walls having a constant heat flux. They found that the Darcy number, porosity, solid-fluid thermal conductivity ratio and Reynolds number all have a significant effect on the results for the different boundary conditions tested, and this was when thermal dispersion and porosity variation are not considered. For a porous matrix with a 
stagnant fluid and a heat flux Lage [19] showed that if the fluid and solid phases are in thermal equilibrium at the boundaries, then they will be in thermal equilibrium throughout the entire domain. Kou and Huang [20] investigated the effect of thermal boundary conditions applied on a vertical annular duct embedded in a porous medium. Teng and Zhao [21] have developed a model that attempts to extend the applicability of the Darcy's law beyond the laminar flow regime as defined by the microscopic Reynolds number of about 10. Optimization of the performance and configuration of porous structures and systems has be the subject of consented research [22-27] whereby analytical models were developed to that end.

Effective cooling of a porous medium made up of solid spheres with internal heat generation rate is dependent on the minimization of the thermal resistance between the porous matrix and the fluid. Conditions under which minimization of the thermal resistance can take place would include the surfaces of the solid spheres and the fluid cooling them being in LTE with one another. The porous matrix under consideration was made up of solid spheres and the determination of the optimum porous properties would essentially mean the determination of the optimum sphere diameter at which the thermal resistance was minimized, for a given porosity. So the aim of the present study was to determine the optimum diameter of same size solid spheres with internal heat generation forming a porous matrix that results in minimum thermal resistance of the porous medium as the spheres are being cooled by air flowing through the porous matrix. This was done for a forced convection laminar flow under steady state conditions.

\section{Analytical model}

The case under investigation in the current study is a cylindrical porous domain with adiabatic walls, saturated with a fluid as shown in Fig. 1. Thermal resistance between the point at a temperature $T_{\max }$ and the point at a temperature $T_{\min }$ as shown in Fig. 1 in a porous medium, as given by Eq. 1, shows that it is directly proportional to the temperature difference between the two points, if the rate of heat transfer is assumed to be constant. In this instance the heat transferred is due to the internal heat generation density, $q^{\prime \prime \prime}$, inside the solid spheres. Here the inlet temperature is defines as the domain minimum temperature, i.e. $T_{\text {inlet }}=T_{\min }$.

$R_{T}=\frac{\left(T_{\max }-T_{\min }\right)}{q}$

From this expression it can be seen that in order to minimize the thermal resistance, all that is required is to minimize the temperature difference. Because the porous medium is made up of air and solid spheres with internal heat generation, the rate of heat transfer is given by $q=(1-\phi) q^{\prime \prime \prime} A L . A$ and $L$ 
are the cross-section area and length of the porous medium respectively, and $\phi$ is the porosity. However this rate of heat transfer must equal the rate of heat removal by the air flowing through the porous medium as given by Eq. 2 given the fact that this analysis is conducted under steady state conditions.

$q=\dot{m} c_{p}\left(T_{\text {outlet }}-T_{\text {min }}\right)$

$c_{p}$ is the heat capacity of the fluid and $T_{\text {outlet }}$ is the area averaged temperature at the porous medium outlet. The mass flow rate of the air flowing with an average velocity $u$ through the porous medium is $\dot{m}=\rho A u$, where $\rho$ is the fluid density. Given that laminar flow is assumed for the air flowing through the porous medium, Darcy's law [28] was used to express the superficial velocity for a unidirectional driven flow as given by Eq. 3 .

$u=\frac{K \Delta P}{\mu L}$

$K, \Delta P$ and $\mu$ are the permeability of the porous medium, the pressure difference between the inlet and outlet and dynamic viscosity of the fluid. Because the solid matrix of the porous medium is made up of solid spheres, the permeability is given by the Carman-Kozeny equation [28] as expressed by Eq. 4.

$$
K=\frac{D^{2} \phi^{3}}{180(1-\phi)^{2}}
$$

$D$ is the diameter of the solid sphere. The temperature difference between the outlet and inlet of the porous medium can be expressed in terms of the diameter of the solid spheres by simply substituting Eqs. 3, 4, into Eq. 2, and rearranging the terms to give Eq. 5.

$T_{\text {outlet }}-T_{\min }=180\left(\frac{1-\phi}{\phi}\right)^{3} \frac{\mu q^{\prime \prime \prime} L^{2}}{\rho C_{p} \Delta P D^{2}}$

The temperature profile of the hottest solid sphere is, $T_{\max }-T_{\text {outlet }}=q^{\prime \prime \prime} D^{2} / 24 k_{s}$, where $k_{s}$ is the solid phase thermal conductivity. When combining the temperature profile of the hottest solid sphere with Eq. 5 results in an expression for the temperature difference between two points in the porous medium having the maximum and minimum temperatures as given by Eq. 6 .

$$
T_{\max }-T_{\min }=\frac{q^{\prime \prime \prime} D^{2}}{24 k_{s}}+180\left(\frac{1-\phi}{\phi}\right)^{3} \frac{\mu q^{\prime \prime \prime} L^{2}}{\rho c_{p} \Delta P D^{2}}
$$


However, the aim of the analysis is the minimization the $T_{\max }-T_{\min }$ with respect to $D$. This results in the optimum sphere diameter as given by Eq. 7 .

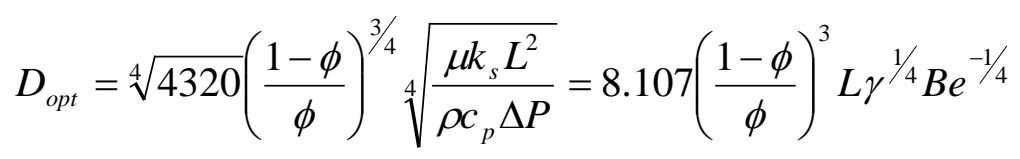

Where $\gamma=k_{s} / k_{f}$ is the solid/fluid thermal conductivity ratio and $B e=\Delta P L^{2} / \alpha \mu$ is the Bejan number [29, 30] representing the dimensionless pressure drop across the porous medium. $\alpha=k_{f} / \rho c_{p}$ is the thermal diffusivity of the fluid.

The minimum temperature difference that corresponds to the optimum diameter is given by Eq. 8.

$$
\left(T_{\max }-T_{\min }\right)_{\min }=5.477\left(\frac{1-\phi}{\phi}\right)^{3 / 2} \frac{q^{\prime \prime \prime} L^{2}}{k_{f}} B e^{-1 / 2} \gamma^{-1 / 2}
$$

When Eq. 8 is substituted into Eq. 1 the result is the minimum thermal resistance as given by Eq. 9.

$$
\left(R_{T}\right)_{\min }=5.477 \frac{(1-\phi)^{1 / 2}}{\phi^{3 / 2}} \frac{L B e^{-1 / 2} \gamma^{-1 / 2}}{A k_{f}}
$$

Eqs. 8 and 9 express the minima temperature difference and thermal resistance in terms of porosity respectively; however it is also useful to express both minima in terms of the optimum diameter by substituting Eq. 7 into Eqs. 8 and 9. The expression for the minimum temperature difference as a function of optimum diameter is given by Eq. 10 and that for the minimum thermal resistance is given by Eq. 11 . Note the disappearance of $q^{\prime \prime \prime}$ and Be in Eq. 11 when expressed in terms of $D_{\text {opt }}$.

$$
\begin{aligned}
& \left(T_{\max }-T_{\min }\right)_{\min }=\frac{q^{\prime \prime \prime} D_{o p t}^{2}}{12 k_{s}} \\
& \left(R_{T}\right)_{\min }=\frac{D_{o p t}^{2}}{12 A L k_{s}(1-\phi)}
\end{aligned}
$$

The optimum diameter, the minimum temperature difference and minimum thermal resistance were all normalized with respect to their respective values at a porosity of $\phi=0.1$. The normalized optimum diameter, $\psi_{\text {opt }}$, the minimum temperature difference, $\theta_{\min }$ and minimum thermal resistance, $\eta_{\min }$ are given by Eqs. 12, 13 and 14 respectively. 
$\psi_{\text {opt }}=\frac{D_{o p t}}{\left(D_{o p t}\right)_{\phi=0.1}}=\frac{\left(\frac{1-\phi}{\phi}\right)^{3 / 4}}{\left(\frac{1-\phi}{\phi}\right)_{\varphi=0.1}^{3 / 4}}$

$\theta_{\min }=\frac{\left(\frac{1-\phi}{\phi}\right)^{3 / 2}}{\left(\frac{1-\phi}{\phi}\right)_{\phi=0.1}^{3 / 2}}=\psi_{o p t}^{2}$

$\eta_{\min }=\frac{\left[\frac{(1-\phi)^{1 / 2}}{\phi^{3 / 2}}\right]}{\left[\frac{(1-\phi)^{1 / 2}}{\phi^{3 / 2}}\right]_{\phi=0.1}}=\frac{0.9}{(1-\phi)} \psi_{o p t}^{2}$

\section{Numerical analysis}

The numerical porous medium model in ANSYS Fluent 13 [31] was used to conduct comparative simulations for this study. Unlike the analytical model (Eq. 6) where the porous medium and the heat conduction inside the solid spheres were modelled, the numerical model models only the porous medium. The flow was driven by a unidirectional pressure gradient across the porous medium.

Steady state was assumed and a three-dimensional domain was also assumed. A further assumption made is that the flow was laminar. With all these assumptions in mind, the flow physics in the porous medium is given by Eqs. 15 to 17 .

The continuity Equation for the porous medium is given by:

$\nabla \cdot(\rho u)=0$

The momentum Equation is given by the Darcy's law:

$-\frac{d P}{d x}=\frac{\mu u}{K}$

The energy Equation is given by:

$\rho C_{p} \vec{v} . \nabla T=k_{m} \nabla^{2} T+q_{m}^{\prime \prime \prime}$ 
Where

$k_{m}=\phi k_{f}+(1-\phi) k_{s}$

And

$q_{m}^{\prime \prime \prime}=(1-\phi) q^{\prime \prime \prime}$

The optimum diameter as given by Eq. 7 was used together with the Carman-Kozeny equation (Eq. 4) to compute the viscous resistance to be inserted into the numerical model. The diameter and length of the porous domain were both $2000 \mathrm{~mm}$. The porous medium domain as represented by Fig. 2 was sandwiched between an up-stream and down-stream domains (which are not shown in Fig. 2 for clarity) used for implementing the inlet and outlet conditions because ANSYS Fluent 13 [31] does not allow direct application of inlet and outlet boundaries on porous domains. The up-stream domain was a fluid domain that was made up of an adiabatic circumferential wall boundary with a non-slip condition and a pressure inlet boundary. The third boundary was simple an interface between the domain and the porous domain connected to it. The static pressure on the inlet boundary was set equal to the operating pressure and the temperature was set to be $25^{\circ} \mathrm{C}$. This domain had 8535 hexahedral cells.

The porous domain was made up of an adiabatic non-slip circumferential wall boundary and two interface boundaries connecting it to up-stream and down-stream domains. The domain had 17070 hexahedral cells. The down-stream domain was a fluid domain. The domain had an interface boundary connecting it to the porous domain, a non-slip adiabatic circumferential wall boundary and a pressure outlet boundary. The change in pressure difference was modelled by varying the gauge pressure of the pressure outlet boundary. The domain had 17070 hexahedral cells. The grid independence of the numerical solution was conducted using the temperature difference, $\Delta T=T_{\text {outlet }}-T_{\text {inlet }}$ as the figure of merit. This was conducted for $q^{\prime \prime \prime}=10^{5} \mathrm{~W} / \mathrm{m}^{3}$, $\Delta P=P_{\text {inlet }}-P_{\text {outlet }}=6 \mathrm{kPa}$ and $\phi=0.1$. The relative change metric used to check the change temperature difference from a coarser grid to a finer grid is $\operatorname{simply}\left(\Delta T_{\text {finer }}-\Delta T_{\text {coarser }}\right) / \Delta T_{\text {finer }}$. As can be seen from Table 1 the change from 2460 cells through to 17070 cells is constant. For this reason the 17070 cells were chosen for the numerical simulations since there was no computational time penalty. 
Table 1. Grid independence study using air as the fluid at $\Delta \mathrm{P}=6 \mathrm{kPa}$ and $\phi=0.1$.

\begin{tabular}{ccc}
\hline Number of cells & $\Delta \mathrm{T}\left({ }^{\circ} \mathrm{C}\right)$ & Relative change \\
\hline 1230 & 56.51124 & \\
\hline 2460 & 57.72587 & 0.021 \\
\hline 4920 & 58.33546 & 0.0104 \\
\hline 17070 & 57.73561 & 0.0104 \\
\hline
\end{tabular}

The implicit pressure-based solver was selected, with the superficial velocity for the porous formulation. The PRESTO! [31] discretization scheme was used for the pressure, and the second order upwind schemes were selected for the density, energy and mass discretization.

Two cases were run where the fluid phase was modelled by first using air and then liquid water. An assumption was made that the fluid (except for density of air) and thermal properties of the solid spheres and fluid are constant. The heat generation rate density that comes from the solid phase used in the numerical simulations was $q^{\prime \prime \prime}=10^{5} \mathrm{~W} / \mathrm{m}^{3}$. For the LTE assumption to be valid for the porosity range of $0.1 \leq \phi \leq 0.9$ the thermal conductivities of the fluid and solid phases making up the porous medium have to of the same order of magnitude. For this reason wood properties (which is important in biomass energy systems) with $k_{\mathrm{s}}=0.173 \mathrm{~W} / \mathrm{m} . \mathrm{K}$ and silica/sand properties (which is important in coal bubbling bed boilers systems) with $k_{s}=0.2 \mathrm{~W} / \mathrm{m} . \mathrm{K}$ were used to model the solid phase properties in the ANSYS Fluent 13 porous medium model. The thermal conductivities of air and liquid water are $0.0242 \mathrm{~W} / \mathrm{m} . \mathrm{K}$ and $0.6 \mathrm{~W} / \mathrm{m} . \mathrm{K}$ respectively.

\section{Results and discussion}

From Eq. 7 it follows that the optimum diameter does not depend on the internal heat generation rate from the solid spheres. This suggests that this optimum diameter solution is robust when applied to a porous medium. This same quality of robustness is also observed with respect to the minimum thermal resistance as expressed by Eq. 11 because it too is independent of the internal heat generation rate from the solid spheres. The normalized optimum diameter was evaluated for a porosity range of $0.1 \leq \phi \leq 0.9$, and this is plotted in Fig. 3. Fig. 3 shows that the optimum diameter decreases nonlinearly with increasing porosity.

The comparison between the results from Eq.13 and those from the normalized ANSYS Fluent 13 (for both air and liquid water) for the normalized minimum temperature difference with respect to the result at a porosity of $\phi=0.1$ is shown in Fig. 4 as functions of normalized optimum diameter. Results for the normalized minimum thermal resistance are also plotted on the same figure. 
Fig. 4 shows that the analytical solution compares very well with numerical results for both air and liquid water. This shows that LTE assumption made in the derivation of the model is valid since the fluid to solid thermal conductivities ratio for both air and liquid water are close to unity or less. From Fig. 4 it can also be seen that the analytical solution much better with the liquid water numerical results than with those of air. This could be as a result of the densities of the liquid water and wood being of the same order of magnitude. This follows from another criterion that was proposed by Whitaker [16] that the thermal diffusivities ratio of the fluid and solid phases should be of the same order of magnitude.

The normalized minimum temperature differences together with the normalized minimum thermal resistance were also plotted against the porosity so as to illustrate the direct relationship between the minima temperature difference and thermal resistance with porosity. As was for Fig. 4 this comparison between the analytical model and the numerical model was done for both air and liquid water, and is plotted in Fig. 5. Again the analytical solution compares very well with both numerical solutions, but compares much better with the liquid water numerical solution because of the thermal diffusivities of the fluid and solid phases being of the same order of magnitude.

\section{Conclusion}

The optimum sphere diameter expression was determined analytically and was found to be independent of the heat generation rate of the solid spheres. This same quality was found to be the same for the corresponding minimum thermal resistance. It can be concluded that the optimum solid sphere diameter and the minimum thermal resistance are both robust when applied to a porous medium where the LTE assumption is valid. The minimum temperature difference analytical solution compared very well to numerical solutions when air and liquid water properties are used for the fluid phase and wood properties are used for the solid phase.

\section{Acknowledgements}

The authors would like to thank the National Research Foundation and the University of Pretoria for providing the financial support and facilities.

\section{References}

[1] B. Alazmi, K. Vafai, Constant wall heat flux boundary conditions in porous media under local thermal non-Equilibrium conditions, International Journal of Heat and Mass Transfer 45 (2002) 30713087. 
[2] D.A. Nield, A.V. Kuznetsov, M. Xiong, Effect of local thermal non-equilibrium on thermally developing forced convection in a porous medium, International Journal of Heat and Mass Transfer 45 (2002) 4949-4955.

[3] Y.-X. Tao, D.M. Gray, Validation of local thermal equilibrium in unsaturated porous media with simultaneous flow and freezing, International Communication in Heat and Mass Transfer 20 (1993) 323-332.

[4] F. Kuwahara, S. Yang, K. Ando, A. Nakayama, Exact solutions for a thermal nonequilibrium model of fluid saturated porous media based on an effective porosity, Journal of Heat Transfer 133 (2011).

[5] A. Amiri, K. Vafai, Analysis of dispersion effects and non-thermal equilibrium, non-Darcian, variable porosity incompressible flow through porous media, International Journal of Heat and Mass Transfer 37 (6) (1994) 939-954.

[6] D.A. Nield, A.V. Kuznetsov, Local thermal nonequilibrium effects in forced convection in a porous medium channel: a conjugate problem, International Journal of Heat and Mass Transfer 42 (1999) 3245-3252.

[7] P. Jiang, Z. Ren, B. Wang, Numerical simulation of forced convection heat transfer in porous plate channels using thermal equilibrium and nonthermal equilibrium models, Numerical Heat Transfer, Part A, 35, 99-113.

[8] S.J. Kim, D. Kim, D.Y. Lee, On the local thermal equilibrium in microchannel heat sinks, International Journal of Heat and Mass Transfer 43 (2000) 1735-1748.

[9] I.S. Shivakumara, J. Lee, K. Vajravelu, A.L. Mamatha, Effects of thermal nonequilibrium and non-uniform temperature gradients on the onset of convection in a heterogeneous porous medium, International Communication in Heat and Mass Transfer 38 (2011) 906-910.

[10] S.J. Kim, S.P. Jang, Effects of the Darcy number, the Prandtl number, and the Reynolds number on local thermal non-equilibrium, International Journal of Heat and Mass Transfer 45 (2002) 38853896.

[11] K. Yang, K. Vafai, Restrictions on the validity of the thermal conditions at the porous-fluid interface - An exact solution, Journal of Heat Transfer 33 (2001).

[12] O.M. Haddad, M.A. Al-Nimr, A.N. Al-Khateeb, Validation of the local thermal equilibrium assumption in natural convection from a vertical plate embedded in porous medium: non-Darcian model, International Journal of Heat and Mass Transfer 47 (2004) 2037-2042. 
[13] M.G. Lloyd, A. Razani, K.J. Kim, Formulation and numerical solution of non-local thermal equilibrium equations for multiple gas/solid porous metal hydride reactors, Journal of Heat Transfer 123 (2001) 520-526.

[14] A.V. Kuznetsov, K. Vafai, Analytical comparison and criteria for heat and mass transfer models in metal hydride packed beds, International Journal of Heat and Mass Transfer 38 ( 15) (1995) 28732884.

[15] B.V.K. Reddy, A. Narasimhan, Heat generation effects in natural convection inside a porous annulus, International Communications in Heat and Mass Transfer 37 (2010) 607-610.

[16] S. Whitaker, Improved Constraints for the Principle of Local Thermal Equilibrium, Industrial Engineering Chemical Resources 30 (5) (1991).

[17] K. Boomsma, D. Poulikakos, On the effective thermal conductivity of a three-dimensional structured fluid-saturated metal foal, International Journal of Heat and Mass Transfer 44 (2001) 827836.

[18] T. Jeng, S. Tzeng, Y. Hung, An analytical study of local thermal equilibrium in porous heat sinks using fin theory, International Journal of Heat and Mass Transfer 49 (2006) 1907-1914.

[19] H. Kou, D. Huang, Fully developed laminar mixed convection through a vertical annular duct filled with porous media, International Communication in Heat and Mass Transfer 24 (1) (1997) 99110.

[20] J.L. Lage, The implications of the thermal equilibrium assumption for surrounding-driven steady conduction within a saturated porous medium layer, International Journal of Heat and Mass Transfer 42 (1999) 477-485.

[21] H. Teng, T.S. Zhao, An extension of Darcy's law to non-stokes flow in porous media, Chemical Engineering Science 55 (2000) 2727-2735.

[22] T. Bello-Ochende, J.P. Meyer, F.U. Ighalo, Combined numerical optimization and constructal theory for the design of microchannel heat sinks, Numerical Heat Transfer, Part A Applications 58 (11) (2010) 882-899.

[23] T. Bello-Ochende, J.P. Meyer, A. Bejan, Constructal mult-scale pin fins, International Journal of Heat and Mass Transfer 53 (13-14) (2010) 2773-2779.

[24] S. Lorente, A. Bejan, Heterogeneous porous media as multiscale structures for maximum flow access, Journal of Applied Physics 100 (2006). 
[25] J.C. Ordonez, A. Bejan, R.S. Cherry, Designed porous media: Optimally nonuniform flow structures connecting one point with more points, International Journal of Thermal Sciences 42 (2003) 857-870.

[26] L. Combelles, S. Lorente, R. Anderson, A. Bejan, Tree-shaped fluid flow and heat storage in a conducting solid, Journal of Applied Physics 111 (2012).

[27] K. Cho, J. Lee, M. Kim, A. Bejan, Vascular design of constructal structures with low flow resistance and nonuniformity, International Journal of Thermal Sciences 49 (2010) 2309-2318.

[28] D.A. Nield, A. Bejan, Convection in porous media, third ed., Springer, New York, 2006, pp. 7.

[29] S. Bhattacharjee, W.L. Grosshandler, The formation of wall jet near a high temperature wall under microgravity environment, ASME HTD 96 (1988) 711-716.

[30] S. Petrescu, Comments on the optimal spacing of parallel plates cooled by forced convection, International Journal of Heat and Mass Transfer 37 (1994) 1283.

[31] www.ansys.com

\section{List of figures}

Fig. 1. Sketch of the porous medium domain.

Fig. 2. Sketch of the numerical porous medium domain with boundary conditions.

Fig. 3. The variation of the normalized optimum diameter with porosity.

Fig. 4. The variation of the normalized minima temperature difference and thermal resistance with normalized optimum sphere diameter.

Fig. 5. The variation of the normalized minima temperature difference and thermal resistance with porosity. 


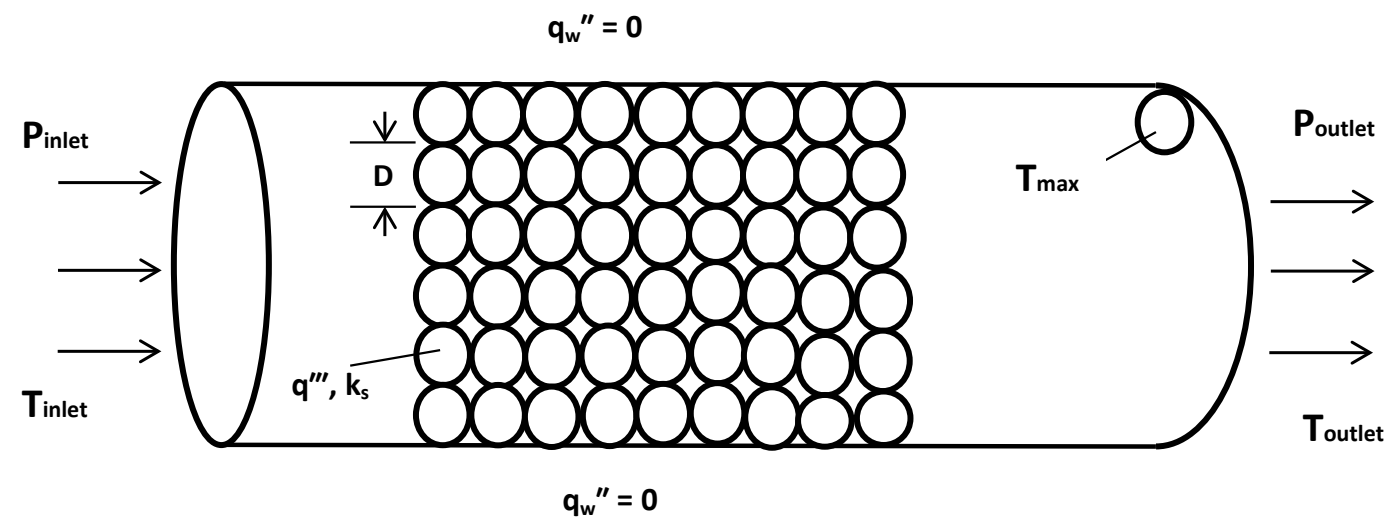

Fig. 1. Sketch of the porous medium domain. 


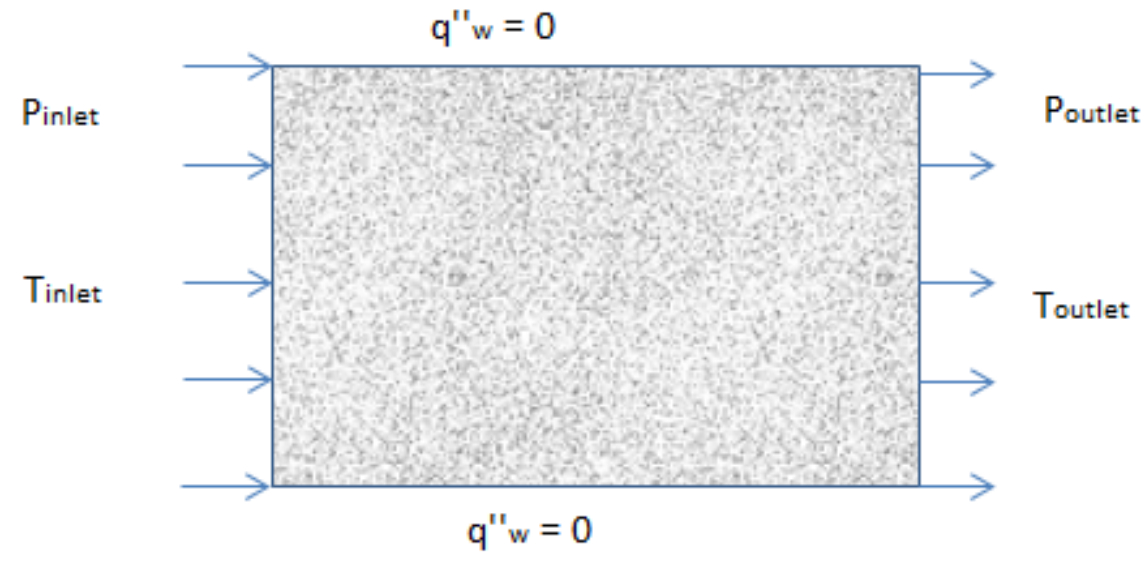

Fig. 2. Sketch of the numerical porous medium domain with boundary conditions. 


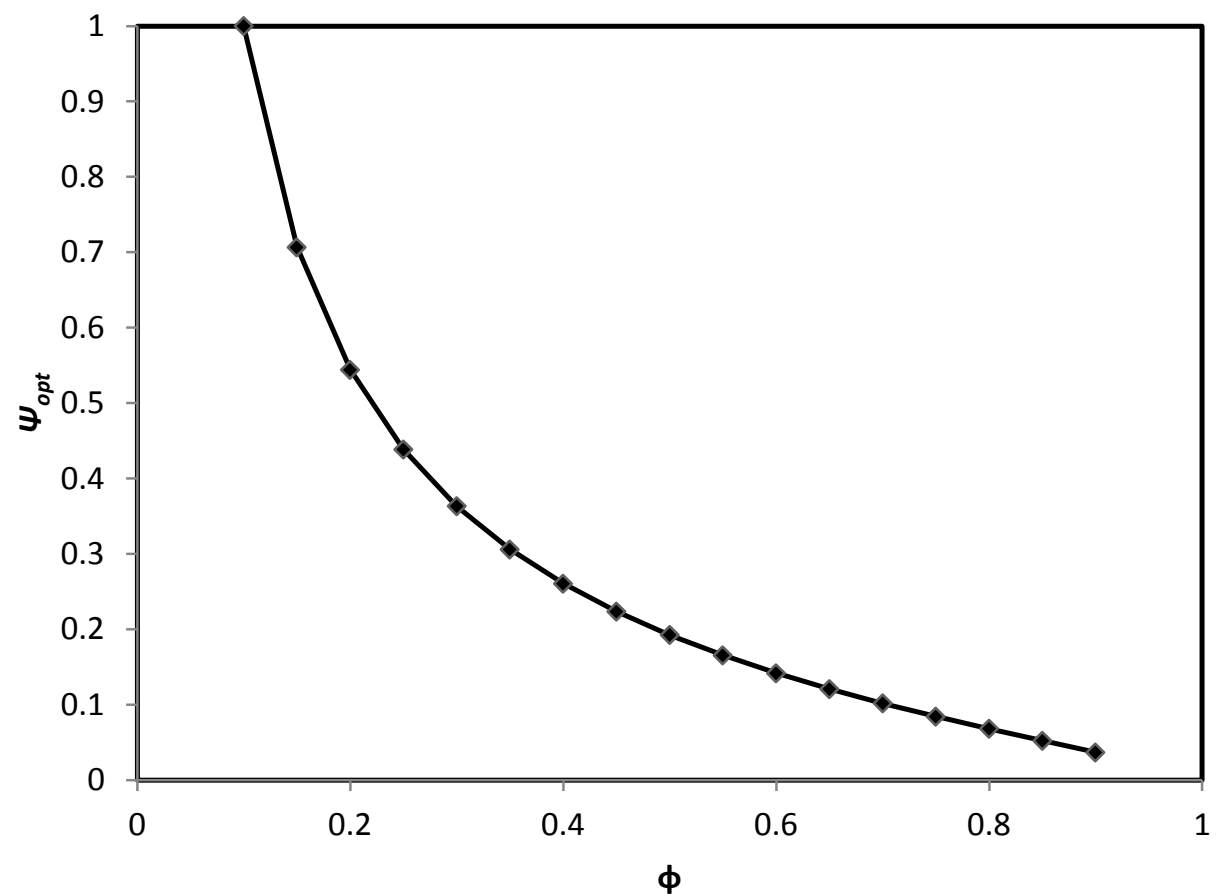

Fig. 3. The variation of the normalized optimum diameter with porosity. 


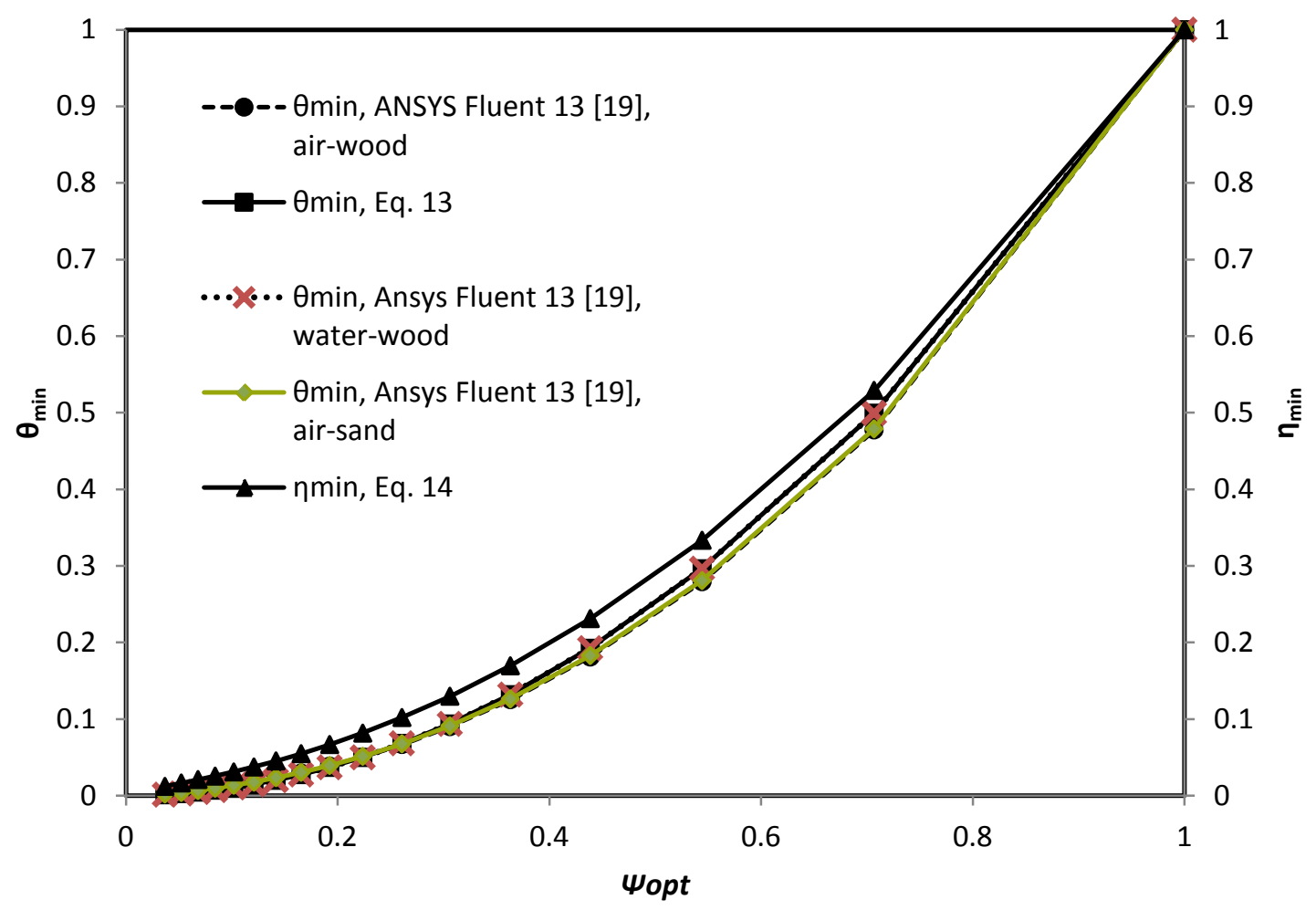

Fig. 4. The variation of the normalized minima temperature difference and thermal resistance with normalized optimum sphere diameter. 


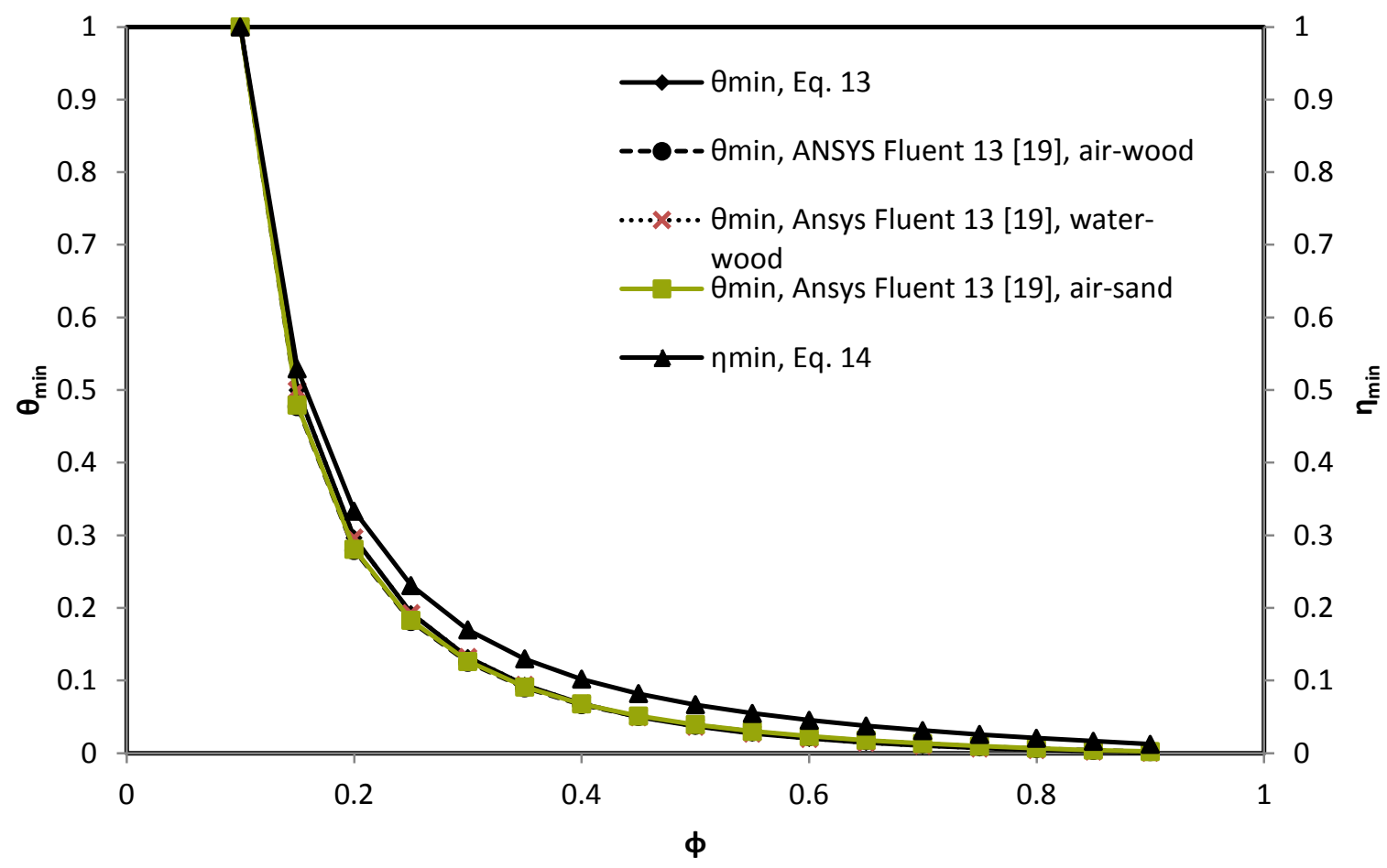

Fig. 5. The variation of the normalized minima temperature difference and thermal resistance with porosity. 